\title{
SPECIFICATION OF INPUT, OUTPUT AND SETTING OPTIONS FOR A BIKE SHARING PLANNING SYSTEM
}

\author{
S. PFOSER \& M. PAJONES \\ Logistikum Steyr, University of Applied Sciences, Upper Austria, Austria.
}

\begin{abstract}
Since the positive effects of active travelling modes are widely proven, the need for propagating them is evident to counteract serious health and environmental problems. The modal share of bicycles can be stimulated by providing sufficient bike sharing systems that allow better access to this active travelling mode. Taking the decision how to introduce bike sharing is in most cases very time consuming and requires a lot of know-how. The Austrian project PlanBiSS is dedicated to the development of methods and strategies which enable an anticipatory planning of such a bike sharing scheme. For this purpose, potential users' requirements regarding input data, output data and setting options have to be determined in a first step in order to obtain knowledge about their expectations towards a bike sharing planning system. We conducted 16 interviews with international and national bike sharing experts as well as planning experts. On the whole, the results represent a basis to develop a planning system that meets the customers' expectations and needs and that facilitates the dissemination of bike sharing systems.

Keywords: bike sharing, planning systems, user expectations.
\end{abstract}

\section{INTRODUCTION}

Bike sharing systems allow their users to gain access to bicycles in public areas. These schemes are characterized by self-service systems that can be used for short-term and oneway rental of bikes. A user can take a bike from one location in the defined network and return it to any other location. Although so far no standardized definition of bike sharing exists [1], it can be clearly differentiated from conventional bike rental systems that are lacking the above described characteristics and that are rather focused on tourism and recreational activities [2, 3]. Another difference is that bicycle usage in bike sharing systems is often limited by time (e.g. using the bikes is free for first $x$ minutes [4]).

The implementation of a bike sharing scheme can actively influence a population's mobility behaviour and change it for the better [5]. For instance, the modal split share of bicycles increased by $1-1.5 \%$ after the introduction of bike sharing in cities like Paris and Barcelona [6]. The focusing of this environmentally friendly and active form of mobility entails a lot of ecological and economic advantages. In most cases, the bicycles are not intended to replace public transport systems but rather to complement them by offering an opportunity to cover the so-called last mile, that is, the distance from the public conveyance station to one's destination.

For planning a new bike sharing scheme there are several handbooks and guides available [7-10]. They suggest first defining the desired density of stations and the covered area as well as some evaluation criteria like vicinity to public transport stations. Most of the time, these decisions are also restricted by budget limitations. The second step is a rough planning of the

This paper is part of the proceedings of the 22nd International Conference on Urban Transport and the Environment (Urban Transport 2016)

www.witconferences.com 
bike sharing station locations. For this purpose, traditional geographic information system (GIS) analysis can be used considering parameters like population density, job density or points of interest. Another option may be crowdsourcing in order to collect proposals from future users of the system [11, 12]. Afterwards, detailed locations will be determined by means of on-site visits and stakeholder dialogues. Finally, the size of the stations is set on the basis of GIS analysis and mobility surveys.

Hence it can be seen that the process of planning the locations of bike sharing stations is rather complex and there are a lot of influencing factors that must be regarded. Manual planning is therefore a tedious and challenging task. Furthermore, planning and operation of bike sharing schemes are mainly separated at the moment. The consolidation of these processes is therefore necessary. For this reason, the Austrian project PlanBiSS aims to develop methods and strategies that could serve as a basis for such a bike sharing planning tool.

\section{METHODOLOGY}

The aim of the present article is to provide basic knowledge about potential users' expectations towards a bike sharing planning tool. Therefore the three main components of such a planning system have been analysed: (1) the input data which is entered into the system, (2) the output of the system, that is, the planning results and finally (3) the setting options to modify planning results.

To determine user requirements, we conducted 16 interviews with different types of experts. Six respondents were planning experts from different Austrian cities. They operate in the fields of traffic planning, especially bicycle traffic planning, as well as urban development and planning. These respondents constituted the main user group of the bike sharing planning tool. Another six respondents can be referred to as 'national bicycle experts'. These interviewees are members of bicycle lobbies or official cycling representatives from different Austrian cities. This means that they have high interest in promoting bicycles as an active mode of transport. For this purpose, their contribution to our research is also relevant. Finally we interviewed four international bike sharing experts who are either involved in the operation of a bike sharing system or who are also doing research on this topic. Their empirical experience is highly valuable for the study.

The interviews were conducted between July and October 2015. All questions were basically asked in two stages: first as an open-ended question so that respondents can contribute new aspects and possibly generate new response options for the question. Afterwards, a list of possible answers was given to the respondent in order to evaluate each of them based on a given scale. For example, we first asked: 'Which input data do you think would be relevant for the bike sharing planning system' (open-ended question). Subsequently we provided a list of possible input data to be evaluated by the respondents (scale question).

The modalities of the interviews varied depending on the type of expert. We conducted detailed personal conversations and phone calls with the planning experts because they represent the main user group and therefore their requirements must be collected most carefully. Some of the bicycle experts are less involved in the issue of bike sharing (e.g. bicycle representatives from small towns), these experts just answered the scale questions in written form and were not asked the open-ended questions. The international bike sharing experts were not met in person; nevertheless they answered the detailed questions telephonically. 


\section{FINDINGS AND DISCUSSION}

As introductory question we asked the interviewees to describe a properly functioning bike sharing system and how they would start to conceptualize such a system. This served for the purpose of confronting them with the topic and identifying themselves with the situation of being responsible for the planning of a bike sharing system. Following this warm-up stage, we started to ask questions about the three main components of a future bike sharing planning system: input data, output data and setting options.

\subsection{Input data}

To obtain the adored output of a planning system, it is usually necessary to fill a system with adequate data. It could be expected that the more detailed the data are, the better the resulting planning results are. However, it must also be kept in mind that a user-friendly application does not require to be fed with a plethora of input data which might possibly even not be feasible to be supplied by the user. For this purpose, we asked the respondents to evaluate input data that are intended to be entered in a bike sharing planning system.

The two-stage survey procedure first included the following open-ended question: 'Which data do you consider as relevant input for a bike sharing planning system?' We received a lot of different and previously unconsidered answers for that question. These responses are:

- Meteorological data

- Traffic safety per road section

- Frequency of use per road section

- Tourist origins (e.g. hotels) and tourist destinations like sights and attractions

- Installations blocking the construction of bike sharing stations

- Statistics about bicycle ownership

- Statistics about bicycle thefts

- Number of bicycle repair shops

After obtaining the suggestions for input data from the respondents, we provided a list with common input data. We derived this list from desktop research and brainstorming. Respondents were asked to assess each item on this list regarding its relevance and regarding its availability. Relevance is defined as the degree of importance for planning a bike sharing scheme on a scale from 1 to 5 (where $1=$ low relevance and $5=$ high relevance). Availability is the degree to which it is possible to provide this data as input on a scale from 1 to 5 (where $1=$ very difficult to provide and $5=$ very easy to provide). The weighted average of each item has been calculated from the values of both dimensions of 'relevance' and 'availability'. These two dimensions are illustrated in Fig. 1.

The two data sets considered as the most relevant input for a bike sharing planning system are topography and information about public transport stations (location and possibly also passenger numbers). At the same time these are the two data sets considered to be the best available.

The quality of existing cycle network is also an important influencing factor for the success of a bike sharing scheme [13]. Respondents also assessed it to be very relevant but, since it is difficult to find objective indicators to evaluate the condition of the bike paths, the availability is evaluated quite low. It is rated to be even more difficult to differentiate the number of public transport guests depending on the time of day. However, this data set is not regarded as very relevant. Data of traffic census is estimated as most subordinate information for a bike sharing planning system. 


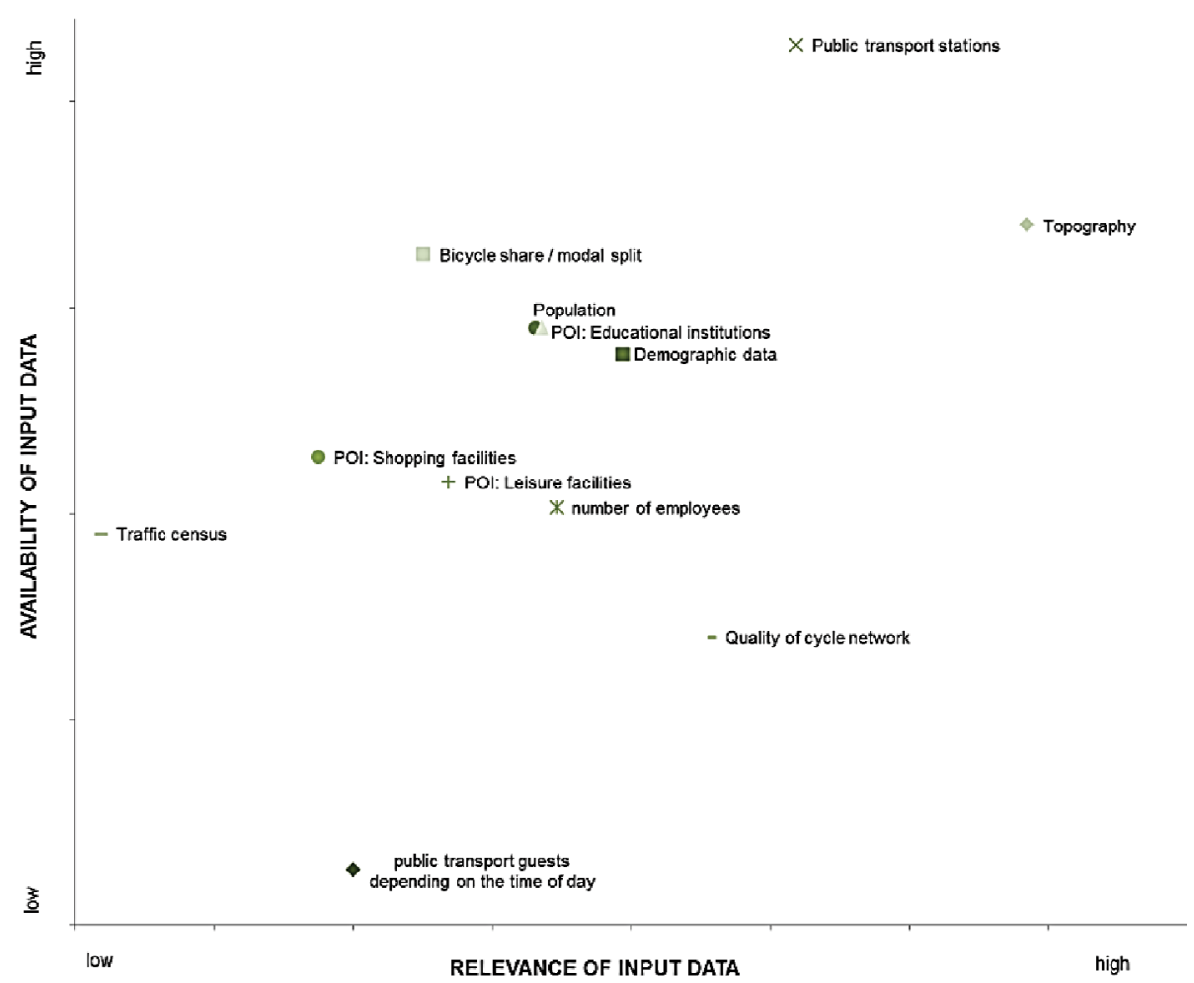

Figure 1: Evaluation of input data for a bike sharing planning system.

The modal split (and the bicycle share in the modal split) is believed to be well available. In this context, some respondents raised the question of how to interpret the bicycle share. The question is whether a high share means that there are many potential users because the residents appreciate riding their bikes or whether a high share means few potential users because they tend to possess and use their own bikes.

Mean values were reached by the remaining input data sets in both dimensions of relevance and availability. These data sets include basic statistic material like population in residential areas, number of employees in industrial areas and demographic data, for example, age. Also various points of interest rank in the midfield like educational institutions, leisure facilities or shopping facilities.

\subsection{Output data}

In order to be able to describe the desired output of a bike sharing planning system, respondents were asked which information they would expect from such a planning software. Again, we compiled a list of possible output data sets and asked respondents to appraise the importance of each item. Importance can be defined as the extent to which it is beneficial and desired that the planning system delivers this output. The degree of importance can be rated on a four-point scale ranging from 'absolutely important', 'important', 'not necessarily important but desirable' to 'not at all important'. Afterwards, the weighted average of each item was calculated. The results are illustrated in Fig. 2. 


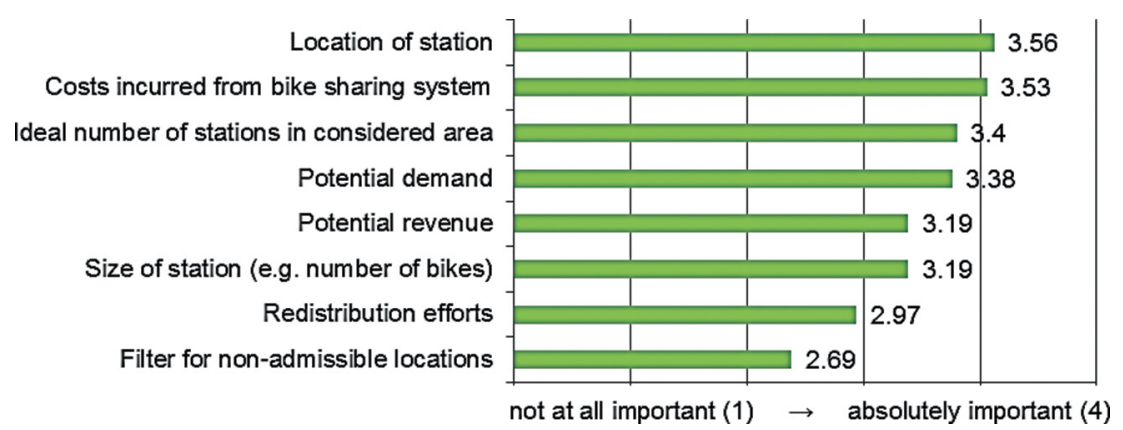

Figure 2: Assessed importance of output data for a bike sharing planning system.

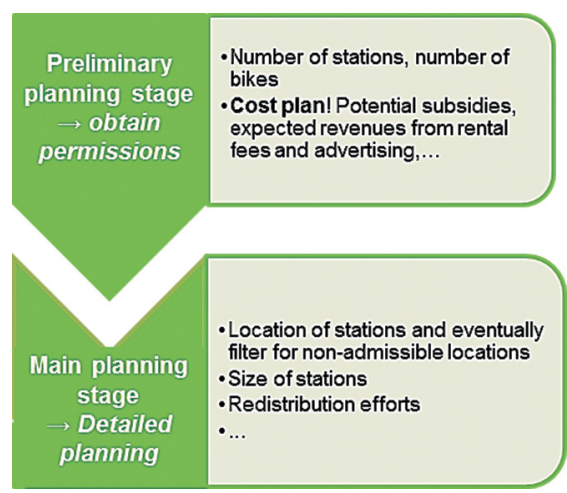

Figure 3: Relevant output data depending on the planning stage.

Within the interviews it was clearly emphasized that the location of bike sharing stations is the most important output of the system. A rough overview of the costs incurred from the bike sharing system (investment costs as well as operating costs) is considered to be of similar importance. Furthermore, the ideal numbers of bike sharing stations in the considered area and the potential demand from the bike sharing system have been rated as important output data, followed by potential revenues and the size of stations. Redistribution efforts have often been assessed as an add-on feature but no necessity. The output data set assessed to be least important is a filter for non-admissible locations. Some respondents stated that this is a task that could also be done manually without much effort.

According to the two-step questioning procedure we asked interviewees once again in an openended way which information they would expect from a bike sharing planning system before the scale question. Respondents suggested that the connection to the cycle network could be provided by the system. Another suggestion was to calculate potential modal shift effects, especially from motorized private transport (like for example in Fishman et al. [14] and Martin \& Shaheen [15]).

It has to be remarked that the importance of the output information partly depends on the planning stage. Consequently, it should be distinguished between the preliminary planning stage and the main planning stage. The main task during the preliminary stage is to obtain necessary permissions. For this reason, only rough plans have to be available in this stage, but it is very important that some first estimations for a cost plan exist. In the main planning stage the details, like for example the definite location of the stations or the size of the stations, are planned (see Fig. 3). 
Finally we also asked if the system should produce a graphical presentation of the planning results. Respondents unanimously emphasized the importance of the visualization of the planning results, especially of the defined locations of bike sharing stations. Significant paths which will be highly frequented according to the predicted demand model could be marked in this map. The marked locations could also be provided with relevant background statistics and information for the implementation of the stations (e.g. is there any infrastructure like power connection available). Ideally, the graphical output is an interactive map where new suggestions of locations are dynamically generated when the input data are changed.

\subsection{Setting options}

In case the user is not satisfied with the planning result, the system needs some settings options to modify the outcome. We prepared a list of possible setting options and asked respondents to judge the usability of each item, again on a four-point scale from 'absolutely important', 'important', 'not necessarily important but desirable' to 'not at all important'. Usability can be defined as the extent to which the setting option is applicable to modify the planning output. Afterwards, the weighted average of each item was calculated. Figure 4 illustrates the results.

Basically those setting options which can hardly be realized in an already implemented and existing bike sharing system have been rated as rather unsuitable. This includes for example the design of bike sharing stations (concreted vs. screwed on the floor, etc.), which will most probably not be changed after the initial investment decision has been realized. Similarly, the design and quality of bicycles might not be changed afterwards. Both of these specifications cannot be decided by a planning system but must rather be determined by human beings. On the contrary, parameters like number, size and density of stations can be calculated by the system. These factors constitute both setting option and output of the planning system. It should be noted that there are two interpretive approaches on the density of stations. On the one hand, the density of stations could refer to the number of stations per unit of area (e.g. number of stations per square meter). On the other hand, the density of stations could also refer to the distance between the stations.

During the interviews, it was sometimes stated that the construction costs of the bike sharing system rather represent an output which results from the other determined specifications.

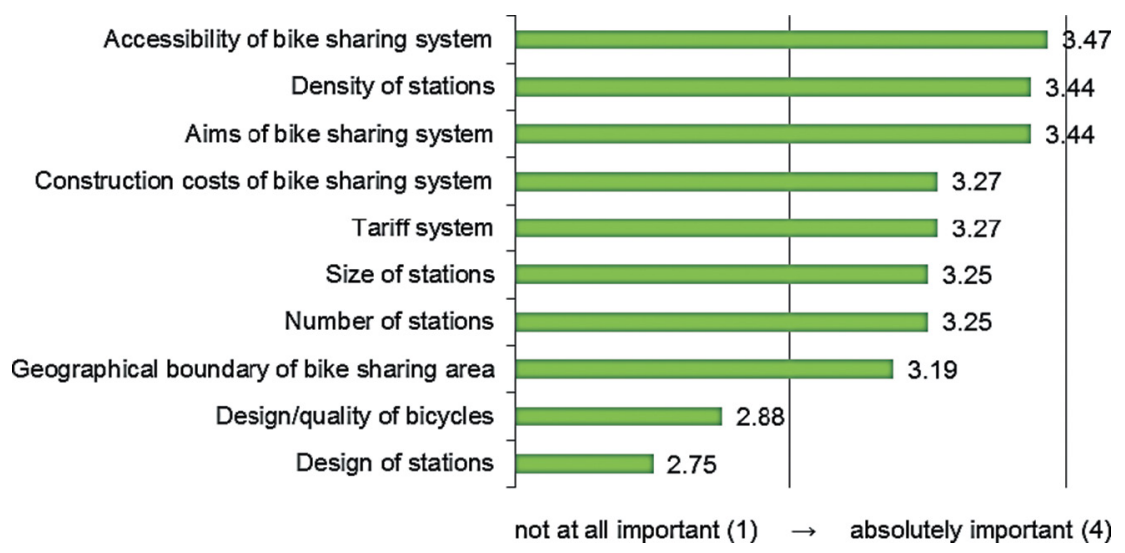

Figure 4: Assessed usability of setting options for a bike sharing planning system. 
Anyway, this function could be practical in case there are specific budget restrictions and it should be investigated which type of bike sharing system could be realized with this budget.

Regarding geographical boundaries, the internal boundaries are especially crucial for the interviewees; this means spaces to be excluded from the planning area like parks or other built-up areas. The possibility to set different aims for the bike sharing system (like reducing motorized private transport, increasing bicycle modal split share, etc.) is acknowledged, because it allows to experiment with the planning result. Accessibility of bike sharing system is rated as the most usable setting option. Accessibility implies the spatial as well as the systemic accessibility, which means the bike sharing stations are spatially well reachable and the process of renting the bikes is also simple.

When asking the open-ended question about setting options, we did not receive any notable suggestions additional to the already proposed list of setting options.

\section{CONCLUSION}

This article gave an overview of bike sharing and traffic planning experts' expectations towards the input, output and setting options of a tool which supports them in planning the implementation and operation of a bike sharing system. The interviews helped to identify which of the proposed data sets are of utterly high importance for potential users and which of them are desirable but not indispensable. Moreover, there is a first estimation of how feasible it is to provide the specified input data since this significantly determines usability of the later developed planning system. Finally, we were also able to describe the required content of the output.

The next step of the project will be to define a data catalogue which brings together and describes all data sets necessary for developing planning algorithms. The obtained interview results serve as a basis for this. The planning algorithms are intended to be used in a followup project to create an automated bike sharing planning tool with a user-friendly interface. Many respondents were also highly interested in receiving the results of the study, so it can be assumed that there will be demand for the tool.

\section{ACKNOWLEDGEMENTS}

This work was accomplished within the project PlanBiSS funded by the Austrian Ministry of Transport within their programme 'Mobilität der Zukunft' (project number 849028). The authors would like to acknowledge the cooperation with AIT Austrian Institute of Technology GmbH, Technische Universität Wien and Rosinak \& Partner ZT GmbH.

\section{REFERENCES}

[1] Büttner, J., Optimising bike sharing in European Cities. Presentation in Milan, 17 September 2010, available at. http://www.obis.pswe.org/palio/html.wmedia? Instance=obis\&_Connector=data\&_ID=444\&_CheckSum=-820062346 $($ accessed 11 February 2016).

[2] Shaheen, S.A., Guzman, S. \& Zhang, H., Bikesharing in Europe, the Americas, and Asia. Transportation Research Record Journal of the Transportation Research Board, 2143, pp. 159-167, 2010. DOI: 10.3141/2143-20.

[3] Zhang, L., Zhang, J., Duan, Z. \& Bryde, D., Sustainable bike-sharing systems: characteristics and commonalities across cases in urban China. Journal of Cleaner Production, 97, pp. 124-133, 2015. DOI: 10.1016/j.jclepro.2014.04.006. 
[4] Lathia, N., Ahmed, S. \& Capra, L., Measuring the impact of opening the London shared bicycle scheme to casual users. Transportation Research Part C: Emerging Technologies, 22, pp. 88-102, 2012. DOI: 10.1016/j.trc.2011.12.004.

[5] Sallis, J.F., Frank, L.D., Saelensc, B.E. \& Kraft, M.K., Active transportation and physical activity: opportunities for collaboration on transportation and public health research. Transportation Research Part A, 38, pp. 249-268, 2004. DOI: 10.1016/j.tra.2003.11.003.

[6] DeMaio, P., Bike-sharing: history, impacts, models of provision, and future. Journal of Public Transportation, 12(4), pp. 41-56, 2009. DOI: 10.5038/2375-0901.12.4.3

[7] Gauthier, A., et al., The Bike-Share Planning Guide. Institute for Transportation and Development Policy: New York, 2013.

[8] Gris Orange Consultant. Bike-Sharing Guide. Transport Canada: Ottawa, 2009.

[9] Toole Design Group \& Pedestrian and Bicycle Information Center. Bike Sharing in the United States: State of the Practice and Guide to Implementation. Washington, DC, 2012.

[10] Schroeder, B., Bicycle Sharing 101: Getting the Wheels Turning. Moonshine Media: Baltimore, 2014.

[11] Daddio, D.W., Maximizing bicycle sharing: an empirical analysis of capital bikeshare usage. PhD thesis, University of North Carolina at Chapel Hill, 2012.

[12] Owen, J., Neita, C. \& Froehlich, J., Crowdsourcing bikeshare transit planning: An empirical investigation of Washington DC and New York City. University of Maryland, available at: https://www.cs.umd.edu/sites/default/files/scholarly_papers/Joseph Owen_Bikeshare.pdf (accessed 11 February 2016).

[13] Fishman, E., Washington, S. \& Haworth, N., Barriers and facilitators to public bicycle scheme use: a qualitative approach. Transportation Research Part F: Traffic Psychology and Behaviour, 15(6), pp. 686-698, 2012. DOI: 10.1016/j.trf.2012.08.002.

[14] Fishman, E., Washington, S. \& Haworth, N., Bike share's impact on car use: Evidence from the United States, Great Britain, and Australia. Transportation Research Part D: Transport and Environment, 31, pp. 13-20, 2014. DOI: 10.1016/j.trd.2014.05.013.

[15] Martin, E.W. \& Shaheen, S.A., Evaluating public transit modal shift dynamics in response to bikesharing: A tale of two U.S. cities. Journal of Transport Geography, 41, pp. 315-324, 2014. DOI: 10.1016/j.jtrangeo.2014.06.026. 